\title{
Effect of Urtica dioica extract on stereological structure of ventral prostate in streptozotocin induced diabetic rats
}

\author{
Gholamreza Hamidian $^{\mathrm{a}}$, Mahsa Zangisheh ${ }^{\mathrm{b}^{*}}$ \\ Department of Basic Science, Faculty of Veterinary Medicine, University of Tabriz, Tabriz, Iran
}

\section{ARTICLE INFO}

\section{* Correspondence to:}

Mahsa Zangisheh

Department of Basic Science, Faculty of Veterinary Medicine, University of Tabriz, Tabriz, Iran e-mail: mahsa_vet@yahoo.com

\section{Keywords:}

Diabetes

Stereology

Urica dioica

Ventral prostate

\section{ABSTRACT}

Diabetes mellitus is a common and important metabolic disorder that can be associated with many functional and structural complications in male sex organs and infertility. Many studies showed that Urtica dioica caused hypoglycemia and prevented diabetes undesirable side effects on the different organs. This study was done to investigate the effects of ethanol extract of Urtica dioica on ventral prostate in streptozotocin induced diabetic rats. In this study, 15 adult male wistar rats were divided into control, diabetic and treatment groups. Hyperglycemia was induced by $50 \mathrm{mg} / \mathrm{kg}$ Streptozotocin (STZ) in diabetic and treatment groups. One week after STZ injection, treatment group received $30 \mathrm{mg} / \mathrm{kg} /$ day Urtica dioica extract by oral gavage for 30 consecutive days. Finally animals were euthanized and ventral prostate were removed and fixed in $10 \%$ neutral buffered formalin. Samples of the ventral prostate were obtained according to the orientator to produce an isotropic uniform random sample. The volumes of the lumen, glandular epithelium and fibromuscular stroma were estimated by multiplying each respective volume density. This study showed that diabetes could lead to structural changes in ventral prostate. Stereological results indicated that volume of lumen and fibromuscular stroma of ventral prostate was significantly increased in diabetic rats but Urtica dioica could inhibit these changes partially. It was also revealed that volume of glandular epithelium was significantly reduced in ventral prostate of diabetic rats but it was significantly increased in treated animals compared to diabetic animals $(\mathrm{p}<0.05)$. It can be concluded that administration of ethanolic extract of Urtica dioicaa can inhibit side effect of diabetes on structure of ventral prostate and has protective effects on its histoarchitecture.

J. Exp. Clin.Med., 2014; 31:132 